Dedhar, Shoukat

[45]

\section{Integrin-linked kinase regulates E-cadherin expression by means of the transcription factor Snail}

\author{
Shoukat Dedhar \\ University of British Columbia and British Columbia Cancer Agency, Vancouver, \\ British Columbia V6H3Z6, Canada
}

The integrin-linked kinase (ILK) is an ankyrin repeat containing serine-threonine protein kinase, which can interact with the cytoplasmic domains of integrin $\beta_{1}$ and $\beta_{3}$ subunits and can be activated by integrins and growth factors. When overexpressed in epithelial cells, ILK inhibits E-cadherin expression, causing an epithelial to mesenchymal transformation. I demonstrate that ILK represses E-cadherin expression by stimulating the expression of Snail, a transcription factor that binds and represses the E-cadherin promoter. Inhibition of ILK in colon cancer cells with a highly selective ILK inhibitor, or dominant-negative ILK, resulted in the inhibition of Snail expression and a concomitant stimulation of expression of Ecadherin. Inhibition of ILK also suppressed $\beta$-catenin/TCF transcriptional activity in $\mathrm{APC}^{-1-}$ colon cancer cells and induced cellular growth arrest as well as growth inhibition of human colon tumors xenografted into SCID mice. Inhibition of ILK may reverse the frequent epithelial-to-mesenchymal transformation associated with tumor invasion and metastasis. ILK is a promising target for the control of cancer progression.

Diehl, Frank

\section{Expression profiling of cervical cancer biopsies using high-resolution dual-label imaging of ${ }^{33} \mathrm{P}$ and ${ }^{3} \mathrm{H}$ on microarrays}

Frank Diehl ${ }^{1}$, Matthias Nees ${ }^{2}$, Heinz Filthuth ${ }^{3}$,

Magnus von Knebel-Doeberitz ${ }^{2} \&$ Jörg Hoheisel ${ }^{1}$

${ }^{1}$ Department of Functional Genome Analysis, German Cancer Research Institute (DKFZ-Heidelberg), Im Neuenheimer Feld 506, 69120 Heidelberg, Germany

${ }^{2}$ Department of Surgery, University of Heidelberg, Im Neuenheimer Feld 110, 69120 Heidelberg, Germany

${ }^{3}$ Zinsser Analytic, Eschborner Landstrasse 135, 60489 Frankfurt, Germany

To identify gene clusters that are involved in the development and progression of cervical cancer we hybridized biopsy material to a custom microarray. Since only small amounts of RNA can be isolated from biopsy material we applied a detection system that is more sensitive than fluorescence detection. The approach combines the advantages of competitive hybridization with the high sensitivity of radioactivity on a microarray platform. Instead of using two fluorescent dyes for labeling we co-hybridized ${ }^{33} \mathrm{P}$ - and ${ }^{3} \mathrm{H}$-labeled probes to our microarray. For the simultaneous detection of the two isotopes we used a new technology that allows the detection of radioactivity in real time with very high resolution $(10 \mu \mathrm{m})$. The unique features of the instrument are a high detection sensitivity for ${ }^{33} \mathrm{P}$ and ${ }^{3} \mathrm{H}$ and an infinite linear range of detection. To select the genes on the array we made use of public gene expression data generated in the past few years (approximately 2,000 clones). In addition to the genes selected from the literature, we added genes obtained from complementary DNA representational difference analysis experiments (approximately 5,000 clones).
Diehn, Maximilian

[47]

From gene expression to the bedside: A high-throughput approach for identifying serum diagnostic markers and immunotherapy targets in human malignancies using DNA microarrays

Maximilian Diehn ${ }^{1}$, Michael B. Eisen², David Botstein ${ }^{2}$ \& Patrick O. Brown ${ }^{1,3}$

${ }^{1}$ Department of Biochemistry, Stanford University School of Medicine, Stanford, California, USA

${ }^{2}$ Department of Genetics, Stanford University School of Medicine, Stanford, California, USA

${ }^{3}$ Howard Hughes Medical Institute and Stanford University School of Medicine, Stanford, California, USA

Tumor-specific membrane-associated and secreted proteins represent a clinically important class of proteins because they have the potential to serve as serum diagnostic markers or immunotherapy targets. We have recently shown that, in addition to allowing gene expression profiling of human tumors, DNA microarrays can be used to identify new membrane-associated and secreted proteins. This can be accomplished by separating messenger RNA species bound to membrane-associated polysomes from other mRNAs and then quantifying the distribution of transcripts in the two fractions by hybridization to DNA microarrays ${ }^{1}$. Using this technique we have now identified approximately 4,000 human gene products that are likely to encode previously unrecognized secreted or membrane proteins. By combining this data set with gene expression profiling data sets from a variety of human malignancies (including those of the breast, liver, kidney, prostate, ovaries, brain and immune system) we have further identified a subset of these 4,000 genes that are candidates for the development of new serum diagnostics and immunotherapies. Many of these markers show more robust tumor-specific gene expression profiles than those currently used at the bedside. We present a paradigm for assigning priority to gene expression data for human malignancies and selecting candidates for downstream analysis that have the highest likelihood of achieving clinical success.

1. Diehn M. et al. Nature Genet. 25, 58-62 (2000).

Divine, Kevin K.

\section{Inactivation of p16 by methylation in human lung adenocarcinoma}

Kevin K. Divine ${ }^{1}$, Frank D. Gilland ${ }^{2}$, Christine A. Stidley ${ }^{3}$, Therese J. Bocklage ${ }^{3}$, Ann Schwartz ${ }^{4}$, Dennis L. Cook ${ }^{1}$ $\&$ Steven A. Belinsky ${ }^{1}$

${ }^{1}$ Lovelace Respiratory Research Institute, Albuquerque, New Mexico, USA

${ }^{2}$ University of Southern California, USA

${ }^{3}$ University of New Mexico, USA

${ }^{4}$ Karmanos Cancer Institute, USA

Adenocarcinoma (AdC) of the central and peripheral lung may arise through distinct molecular mechanisms resulting from differences in exposure of these lung compartments to carcinogen. Inactivation of the p16 gene by aberrant promoter hypermethylation, an epigenetic mechanism, has been implicated in AdC development associated with tobacco. We investigated the frequency of p16 methylation in $\mathrm{AdC}$ from three different populations: smokers, former uranium miners who 